\title{
Visceral Pain and Gastrointestinal Microbiome
}

\author{
Maciej Chichlowski ${ }^{1 *}$ and Colin Rudolph ${ }^{1,2}$ \\ ${ }^{1}$ Mead Johnson Nutrition, Evansville, IN, USA; and ${ }^{2}$ Division of Pediatric Gastroenterology, Hepatology, and Nutrition, Department of \\ Pediatrics, University of California, San Francisco, CA, USA
}

\begin{abstract}
A complex set of interactions between the microbiome, gut and brain modulate responses to visceral pain. These interactions occur at the level of the gastrointestinal mucosa, and via local neural, endocrine or immune activity; as well as by the production of factors transported through the circulatory system, like bacterial metabolites or hormones. Various psychological, infectious and other stressors can disrupt this harmonious relationship and alter both the microbiome and visceral pain responses. There are critical sensitive periods that can impact visceral pain responses in adulthood. In this review we provide a brief background of the intestinal microbiome and emerging concepts of the bidirectional interactions between the microbiome, gut and brain. We also discuss recent work in animal models, and human clinical trials using prebiotics and probiotics that alter the microbiome with resultant alterations in visceral pain responses.
\end{abstract}

(J Neurogastroenterol Motil 2015;21:172-181)

\section{Key Words}

Microbiome; Prebiotics; Probiotics; Visceral pain

\section{Introduction}

Visceral pain resulting from distension, ischemia or inflammation of abdominal viscera is mediated through peripheral pathways and the central nervous system. Factors that modulate each individual's perception of a stimulus as pain are beginning to be unraveled. Among these, emerging data show that there is an interaction between the intestinal microbiome and pathways mediating visceral pain. The absence of gastrointestinal (GI) bacteria, such as which occurs in germ free (GF) mice, is associated with reduced perception of pain following different inflammatory stimuli. ${ }^{1}$ Furthermore, modulation of the intestinal microbiome by administration of various probiotics also has been shown to alter pain responses. ${ }^{2,3}$ This review provides a brief background of the intestinal microbiome and emerging concepts of the bidirectional interactions between the microbiome, gut, and brain. Because changes in early life have been shown to impact lifelong visceral pain responses, further details are provided regarding both development of the microbiome and the impact of early stress on the microbiome. Finally, we discuss recent work in animal models and human clinical trials that show how agents altering the microbiome has potential therapeutic value to modulate visceral pain responses, and suggest that these effects may be

Received: February 16, 2014 Revised: March 10, 2015 Accepted: March 11, 2015

(c) This is an Open Access article distributed under the terms of the Creative Commons Attribution Non-Commercial License (http://creativecommons. org/licenses/by-nc/3.0) which permits unrestricted non-commercial use, distribution, and reproduction in any medium, provided the original work is properly cited.

*Correspondence: Maciej Chichlowski, PhD

Mead Johnson Nutrition, 2400 W. Lloyd Expressway, Evansville IN 47721, USA

Tel: +1-916-943-6713, E-mail: maciej.chichlowski@mjn.com

Financial support: This study was financially supported by Mead Johnson Nutrition.

Conflicts of interest: Maciej Chichlowski and Colin Rudolph are employees of Mead Johnson Nutrition.

Author contributions: Maciej Chichlowski and Colin Rudolph contributed equally to collecting and interpreting the data as well as drafting this manuscript.

ORCID: Maciej Chichlowski, http://orcid.org/0000-0002-6004-2195; Colin Rudolph, http://orcid.org/0000-0002-2842-1224. 
helpful in prevention of long-term sequelae of pain experienced early in development.

\section{The Intestinal Microbiome}

Modern molecular techniques have allowed a deeper understanding of the development, metabolic activity and potential interactions between the GI microbiome and the host organism. ${ }^{4}$ In the adult the gut microbiota is composed of $10^{13}-10^{14}$ microorganisms, and contains 100 times as many genes as the whole human genome. ${ }^{5}$ The composition of the microbiota varies according to the region of the GI tract. Gram-positive facultative anaerobic bacteria predominate in the proximal small intestine, while gram-negative anaerobes do so in the distal small intestine. In the colon, obligate anaerobes outnumber facultative anaerobes. The microbiota produce a large range of metabolic substances that vary among the different bacteria composing each individual's microbiome, and with the substrates supplied in the diet. ${ }^{6}$ These include endogenous vitamins such as folate and biotin; short chain fatty acids such as propionate, butyrate, and acetate, and a variety of neuroactive metabolites such as serotonin and gammabutyric acid. ${ }^{7}$

\section{Development of the Gastrointestinal Microbiome}

The gut is colonized by bacteria from the moment of birth, rapidly achieving concentrations of up to $10^{12}$ organisms per gram of luminal contents in the colon. ${ }^{8}$ Many factors influence the composition of the infant gut microbiota and the potential functional outcomes following colonization (reviewed in Borre et $\mathrm{al}^{9}$ ). For example, infants born by vaginal delivery are colonized by the maternal microbiome (eg, fecal and vaginal bacteria), whereas infants born by cesarean delivery are exposed to different commensals from the skin and hospital environment. ${ }^{10-12}$ Infant diet also impacts the microbiome such that the diet of the breast fed infant differs from those fed with infant formula. ${ }^{13}$

The gut microbiome contributes to the early programming of epithelial barrier function, angiogenesis, and innate and host immune function. ${ }^{14}$ Delayed intestinal bacterial colonization such as occurs in GF mice can have prolonged, lifelong influence on the immune system with aberrant development of the innate immune system and altered immunoregulatory responses later in life. ${ }^{15-17}$ Similarly, in humans, alterations in the pattern of GI colonization are thought to have long-term consequences on immune function.
Children born by caesarean section or who receive antibiotics during infancy have a higher incidence of allergy. ${ }^{18,19}$ The infant transitions towards a more typical adult microbiome with weaning and by 2 years of age the microbiome is similar to that of adults. ${ }^{20}$

\section{Microbiota-Gut-Brain Axis}

The microbiome can influence both peripheral and central neurological activity by a variety of mechanisms (Figure). It has long been recognized that the presence of bacteria within the GI lumen can influence myoelectric activity. The introduction of bacteria to GF rats increases slow wave frequency and promotes aboral propagation of the migrating motor complex. ${ }^{21}$ Bacterialderived endotoxins, such as lipopolysaccharides, alter gut motility by activating the enteric nervous system, and bacterial derived peptides such as formyl-methionyl-leucyl-phenalanine have been shown to stimulate primary afferent nerves. ${ }^{21}$ Some bacterial derived products may have direct effects on enteric nerves but epithelial cell interactions may also be required for activity. For example, microvesicles derived from Bacteroides fragilis increase excitability of myenteric intrinsic primary afferent neurons when applied to the mucosal surface but have no effect when applied directly to the myenteric plexus neurons indicating that bacteria or their components may communicate with local neurons indirectly through signals generated in the epithelium. ${ }^{22}$

Bacterial products have also been shown to have profound effects on behavior. In mice, maternal immune activation (MIA) with the viral mimic poly I:C (polyinosinic:polycytidylic acid) during pregnancy yields offsprings that exhibit behavioral symptoms of autism. Treatment with $B$. fragilis ameliorates some of these behavioral changes in the offspring. ${ }^{23}$ Identification of changes in the serum metabolome associated with such treatment led to the identification of several bacterial metabolites that could explain this effect. Administration of one candidate bacterial metabolite, 4-ethylphenylsulfate to naive mice induced anxiety-like behaviors, similar to those in the mice from the MIA mothers. This suggests that circulating bacterial metabolites may mediate changes in behavioral state.

The brain may also modulate the composition of the gut microbiota. Restraint stress is shown to disrupt the microbiome in mice leading to an increase in colonization by Citrobacter rodentium, possibly by altering the local mucosal microenvironment, so that bacterial adherence patterns change. ${ }^{24,25}$ More recently, it was demonstrated that exposure to as little as 2 hours of a social stressor (placement of a young $\mathrm{C} 57 \mathrm{BL} / 6$ mouse in a cage with an 


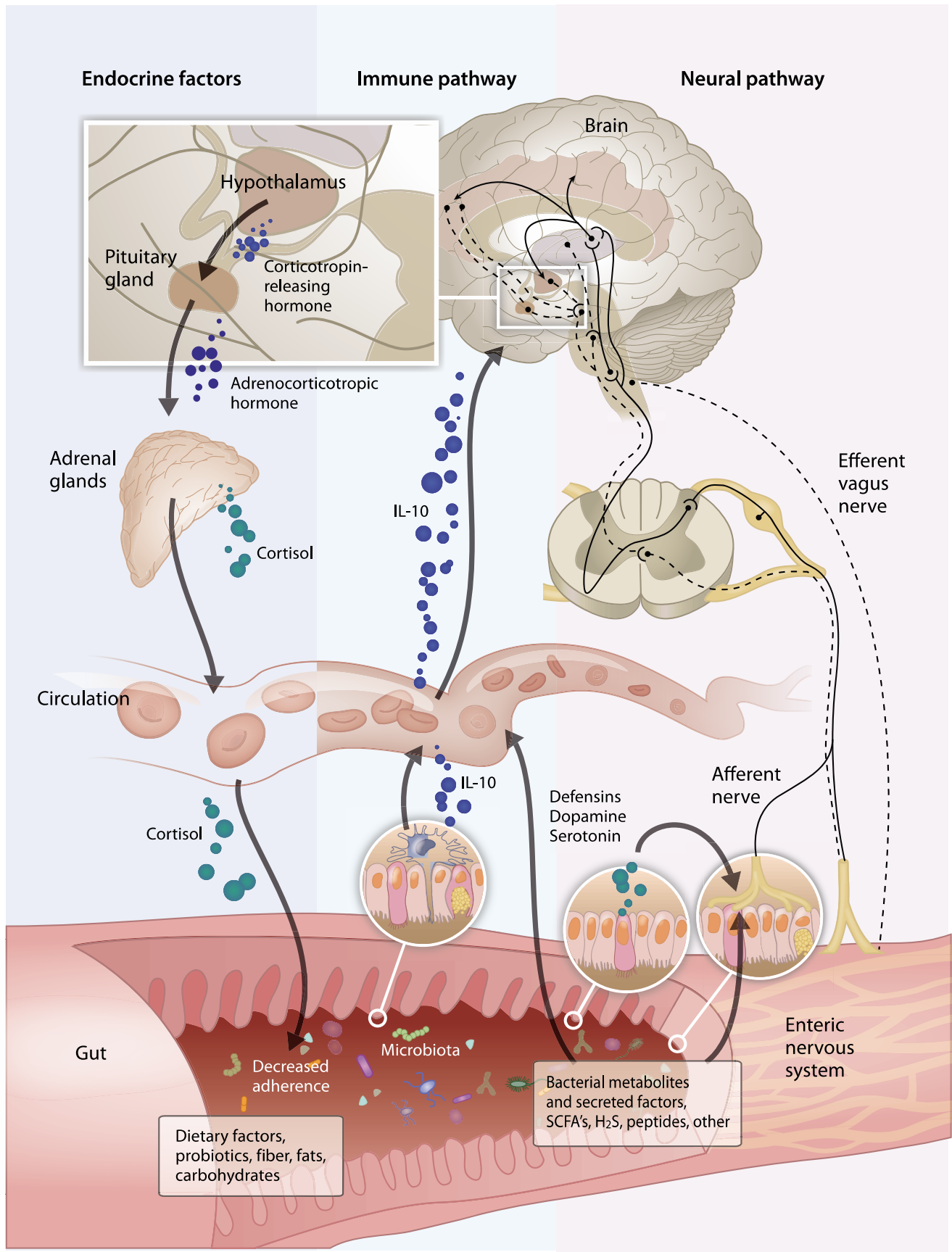

Figure. This figure illustrates the interactions between microbiome, gut and brain which modulate responses to visceral pain. These interactions occur at the level of the gastrointestinal mucosa, and via local neural, endocrine or immune activity, as well as by the production of factors transported through the circulatory system, like bacterial metabolites or hormones. Endocrine Factors: in germ free mice, chemical changes were associated with an exaggerated hypothalamic pituitary stress response, eg, elevation of plasma adrenocorticotropic hormone and corticosterone. Immune Pathway: the intestinal microbiota secretes factors that alter the mucosal permeability and macrophage release of IL-10. Neural Pathway: while the visceral pain results from the activation of nociceptors in the abdominal viscera, the visceral nociceptive afferent fibers further project onto spinal nociceptive neurons located in the superficial laminae, the lateral neck of the dorsal horn and lamina X of spinal cord that convey information to supraspinal centers. Gut: the microbiota produces a large range of metabolites which include short chain fatty acids (SCFA) and a variety of neuroactive metabolites such as serotonin. It is hypothesized that hydrogen and hydrogen sulfide may be bacterial metabolites responsible for visceral hypersensitivity. For example, in mice, hydrogen sulfide directly triggers visceral nociceptive behavior through sensitization and activation of T-type channels in the primary afferents. 
aggressive older CD-1 mouse) altered composition of the colonic mucosa-associated microbiota. ${ }^{26}$ The mechanisms causing these changes are yet to be elucidated but such a rapid change is likely mediated by changes in bacterial adhesion, mediated by either endocrine or neuronal effects on the GI mucosa (Figure).

In summary, there are complex interactions between the microbiome, gut and brain increasingly discussed in the context of the "microbiota-gut-brain axis." Bidirectional communications occur where the microbiota influences the host and the host alters microbiota composition. These interactions are modulated at the level of the GI mucosa, and via the modulation of local neural, endocrine, or immune activity; as well as by the production of factors transported through the circulatory system (Figure).

\section{Microbiome and Neurodevelopment}

The early neonatal period is a critical time for the development of the nervous system, including the enteric nervous system. ${ }^{27,28}$ Recent studies comparing the development of the enteric nervous system in GF mice and specific pathogen-free mice suggest that the intestinal microbiota plays an important role in shaping this process. ${ }^{9}$ In GF mice the myenteric plexus of the jejunum and ileum show a decrease in nerve density and the number of neuronal cell bodies per ganglion but an increase in nitrergic neurons. The frequency and amplitude of muscle contractions also were fewer in GF mice. ${ }^{29}$ GF mice also differ from conventional mice in the expression of brain-derived neurotrophic factor in the cortex and hippocampus, and synaptophysin and post-synaptic density protein-95 in the striatum, relative to specific pathogen free mice. These quantifiable chemical changes were associated with an exaggerated hypothalamic pituitary stress response (elevation of plasma adrenocorticotropic hormone and corticosterone) in the GF mice. Reconstitution with Bifidobacterium infantis during early development, but not later in development, lessened these differences. In contrast, the reconstitution with Escherichia coli enhanced the stress response. ${ }^{30,31}$ This suggests that during the early neonatal period, there is a critical window at which the microbial colonization of the GI tract influences the development of both the peripheral and central nervous system.

As in older animals, stressor exposure early in life alters the types and abundance of bacteria found in the intestines. The stress of separating infant monkeys from their mothers reduces the number of total fecal lactobacilli. ${ }^{32}$ Similarly, separation of rat pups from their mothers during the first 14 days of life alters the GI microbiome. ${ }^{33}$ These changes in microbiome may be asso- ciated with exaggerated visceral pain responses that persist through adulthood in rats following maternal separation. ${ }^{33}$

\section{Visceral Pain During Development Alter Pain Responses in Adulthood}

Visceral pain results from the activation of nociceptors in the abdominal viscera. Visceral nociceptive afferent fibers project onto spinal nociceptive neurons located in the superficial laminae, the lateral neck of the dorsal horn and lamina $\mathrm{X}$ of spinal cord that convey information to supraspinal centers (Figure). ${ }^{34}$ Brain regions that generate pain perception and modulate response to painful stimuli through descending inhibition at the spinal level include the cingulate cortex, medial thalamus, amygdala, hypothalamus, periaqueductal gray, and the solitary tract. ${ }^{35}$

Visceral hypersensitivity refers to a decreased pain threshold following nociceptor activation, or to an exaggerated response to the painful stimulus. The mechanisms underlying this increased responsiveness might include (1) sensitization of primary sensory afferents innervating the viscera, (2) hyperexcitability of spinal ascending neurons (central sensitization) receiving synaptic input from the viscera, (3) dysregulation of descending pathways that modulate spinal nociceptive transmission, and (4) changes in the central perception of a painful stimulus. ${ }^{36}$

As with the development of the microbiome, the early neonatal period is a key critical sensitive period for the development of the neural nociceptive pathways and sensory nerves. Stress experienced in early life triggers long-term changes in visceral sensitivity to noxious stimuli. A well-established experimental model of visceral hypersensitivity utilizes moderate periods of maternal separation as a stressor in neonatal rats. As adults, the stressed animals demonstrate increased visceral hypersensitivity as assessed by the response to colorectal distension. ${ }^{37}$ Similar findings of visceral hypersensitivity are observed in adult rats following recurrent neonatal somatic pain such as that induced by acid injection into a muscle or nasogastric suctioning. The adult pain hypersensitivity can be blocked by preemptive administration of glutamate receptor antagonists (given at the time of pain induction) in the case of acid injection, ${ }^{38}$ or corticotrophin releasing factor 1 antagonist in case of nasogastric suctioning. ${ }^{39}$ These findings and many others indicate that the changes in visceral pain responses induced during infancy can be modified by changing the neurochemical milieu during the painful experience. 


\section{Alterations in the Microbiome and Visceral Pain Responses in Animal Models}

Animal models have been useful to demonstrate potential mechanisms by which the microbiome can modulate visceral pain responses. $^{2,3,40-43}$ In GF mice, contact with commensal microbiota is necessary for mice to develop pain sensitivity, possibly in a toll-like receptor (TLR)-dependent manner. ${ }^{1}$ Inflammatory hyper nociception induced by diverse stimuli, including lipopolysaccharide (LPS) and Interleukin (IL)-1 $\beta$ is reduced in GF mice. The intestinal microbiota secrete factors that among other actions, alter the mucosal permeability, macrophage release of IL-10, T-regulatory cell differentiation, dendritic cell release of transforming growth factor beta (TGF- $\beta$ ) and other cytokines, and induction of Th17 cells. ${ }^{44}$ It is known that immune cell secretions including cytokines alter visceral pain responses. For example, administration of the cytokine inhibitor, diacerein, reduces acetic acid-induced nociception in mice, while inhibiting production of IL- $1 \beta$ and tumor necrosis factor alpha (TNF- $\alpha){ }^{45}$ Thus, it is reasonable to assume that some of the effects of the microbiota on visceral pain responses are mediated via microbiotaneuroimmune interactions. Administration of antibiotics to mice for 2 weeks attenuated the visceral pain-related responses to intraperitoneal acetic acid or intra-colonic capsaicin. ${ }^{46}$ These changes were associated with increased levels of secretory-IgA, upregulation of the antimicrobial peptide resistin-like molecule beta and TLR5, and upregulation of the cannabinoid 1 receptor and downregulation of the mu-opioid receptor. Thus, alterations in the gut microbiota are associated with changes in a variety of pain-related pathways. Changes in mucosal permeability, mucosal immune system composition and the microbiome populations are also described in humans with irritable bowel syndrome (IBS) suggesting that microbiota-neuroimmune interactions play a key role in some visceral pain syndromes. ${ }^{47}$

Administration of probiotic bacteria has impact on neuronal excitability and motility in animal models. Administration of Lactobacillus rhamnosus increases enteric neuron excitability and modulates colonic motility in rodents. ${ }^{48,49}$ Moreover, it has been suggested that Lactobacillus farciminis exerts antinociceptive effects by altering central sensitization. ${ }^{50}$ McKernan and colleagues ${ }^{43}$ compared the efficacy of 3 probiotics (Lactobacillus salivarius UCC118, B. infantis 35624, and Bifidobacterium breve UCC2003) on the abdominal response to colorectal distension using visceral normosensitive rats (Spargue-Daweley) and visceral hypersensitive rats (Wistar Kyoto). In that study, B. infantis 35624 reduced the colorectal distension-induced pain behavior in both rat strains. Interestingly, in a clinical trial, comparing an 8 week treatment by $B$. infantis 35624 and $L$. salivarius UCC118 in IBS patients, only $B$. infantis 35624-treated patients experienced a reduction in composite and individual scores for abdominal pain and discomfort, bloating and distension. ${ }^{51}$ Lactobacillus spp. was implicated in the modulation of visceral pain; for example, it has been shown that excitability of dorsal root ganglia in response to colorectal distension is prevented by $L$. rhamnosus treatment. ${ }^{42}$ In another study, treatment with Lactobacillus species upregulated cannabinoid receptor 2 expression in rats and mice, leading to the induction of visceral analgesia. ${ }^{3}$

Bravo et $\mathrm{al}^{52}$ have reported that the vagus nerve is possibly the major modulatory nervous pathway between the probiotic bacteria in the gut and the brain (Figure). Other studies have shown that anxiety-related behavior was reduced after probiotic treatment, as long as vagus nerve integrity was maintained. ${ }^{52,53}$ In the study by Bercik and colleagues ${ }^{53}$ mice with experimentally induced chronic colitis showed anxiety-like behavior. Treatment with Bifidobacterium longum abolished such behavior. However, the anxiolytic effect of $B$. longum was absent in vagotomized mice, suggesting that the effect was transmitted to the central nervous system by activating vagal pathways at the level of the enteric nervous system. In addition, a recent experimental study demonstrated that $L$. rhamnosus effects on emotional behavior and the central gamma-aminobutyric acid (GABA)-ergic system in mice are regulated via the vagus nerve. ${ }^{52}$ Stress causes a release of central corticotrophin-releasing factor which stimulates the vagal efferents that alter intestinal permeability and gut motor function. ${ }^{54,55}$ These observations support a view that the gut microbiota play a fundamental role in modulating visceral pain responses via neural, immune, and endocrine interactions (Figure). ${ }^{1-3}$

\section{Altered Microbiome During Early Life Critical Sensitive Periods Impacts Visceral Pain in Adulthood}

As discussed above, there are critical sensitive periods that can impact visceral pain responses in adulthood. Antibiotics profoundly alter the GI microbiota, and different antibiotics may result in dissimilar patterns of dysbiosis. Aguilera and colleagues ${ }^{56}$ studied the impact of broad spectrum antibiotics (bacitracin/neomycin) on the visceral hyperalgesia observed after psychological stress induced by water avoidance in mice. Antibiotic treatment 
was associated with a decrease in luminal bacteria, and a dysbiosis with increased bacterial adherence. There was an upregulation of cannnabinoid receptors 1 and 2, and a reduction in visceral hypersensitivity suggesting one possible mechanism by which microbial alterations can blunt the visceral pain response. In nonstressed animals, antibiotics had no effects on visceral pain responses. Verdú et $\mathrm{al}^{2}$ demonstrated that in rodents, antibiotic treatment was associated with increased inflammation in the colon which was also associated with increased substance $\mathrm{P}$ immunoreactivity, and visceral hypersensitivity. Disruption of the microbiome during early life by administration of vancomycin for postnatal day 4-13 led to visceral hypersensitivity to colorectal distension in the adult rats, even though the dysbiosis had resolved. ${ }^{57}$ Interestingly, vancomycin treatment did not impact cognitive or anxiety-related behaviors, growth or other parameters in the adult animals. Recently, Kannampali and colleagues ${ }^{58}$ used the rat chronic visceral hypersensitivity model to test the effect of probiotics and prebiotics on ameliorating the severity of neonatal stress-induced hypersensitivity. In that model, introduction of zymosan into the colon during the neonatal period produces short-term inflammation and subsequent long-term colonic hypersensitivity. Those researchers reported that L. rhamnosus ATCC 53103 (Lactobacillus GG; LGG) attenuates chronic visceral hypersensitivity after animals were exposed to early life painful stimulus. The prebiotic mix (galactooligosaccharides and polydextrose) also expressed significant analgesic effect, but to a less extent compared to LGG. Furthermore, LGG was found to alter the levels of brain neurotransmitters, like serotonin, noradrenaline, and dopamine which are known to be involved in pain modulation. ${ }^{59}$ In a study by Kamiya et al, ${ }^{60}$ treatment with live and killed Lactobacillus reuteri prevented the pain response to colorectal distension by decreasing of the dorsal root ganglion single unit activity to distension. In another study, $L$. reuteri was found to reduce sensation of pain via the enteric nerve in a model of visceral pain induced by colorectal distension. ${ }^{60} \mathrm{~A}$ decrease of normal visceral perception and chronic colonic hypersensitivity, elicited by butyrate was also observed after an oral treatment by L. acidophilus NCFM. ${ }^{3}$

\section{Alterations of the Microbiome in Visceral Pain Disorders}

IBS is characterized by chronic abdominal pain and discomfort. Growing evidences suggest that IBS patients have a dysbiotic intestinal microbiota. ${ }^{61,62}$ Approximately $8 \%$ of children experience recurrent functional abdominal pain and about $61 \%$ of these chil- dren continue to report abdominal pain or IBS. In childhood, recurrent abdominal pain (RAP) consists of pain symptoms similar and often indistinguishable from those in IBS suggesting that there are similar underlying pathophysiologies. ${ }^{63}$ Although infantile colic has not been linked directly to visceral pain, it is generally assumed that the correlation exists.

There are several possible etiologies for the sensory abnormalities in IBS, RAP and colic such as the receptors in the gut wall, the primary sensory afferent neurons, the spinal cord and the brain itself. ${ }^{64}$ The developmental timing at which these alterations in responsiveness appear (ie, during infancy, childhood, and adulthood) remains unclear but many IBS patients have histories of early life stressors. ${ }^{65}$ The link between IBS and increased intestinal sensitivity has been previously described ${ }^{66}$ and visceral hypersensitivity and visceral pain are the important pathophysiological factors in IBS. These factors are abnormal in patients suffering from IBS and about $35 \%$ of the IBS patients have chronic pelvic pain. ${ }^{67}$ Mayer et $\mathrm{al}^{68}$ reported that IBS patients exhibit increased activation of brain regions that are linked to perception of rectal distension and Mertz et $\mathrm{al}^{69}$ showed differences in brain activation patterns in response to a painful rectal stimulus in IBS patients compared to controls. Many studies have also shown that those with IBS have amplified visceral sensitivity in response to stress $^{70}$ or food intake. ${ }^{71}$ For example, patients with IBS had enhanced modulation of visceral perception when subjected to auditory or mental stress. ${ }^{72,73}$ Patients with IBS frequently have accompanying psychological disorders, such as anxiety or depression, and those with psychological stress are more likely to develop post-infectious IBS. Abnormal bowel gas accumulation may be linked to bacterial metabolism and abdominal bloating and may be further associated with visceral hypersensitivity and the impaired gas handling observed in some IBS patients. ${ }^{74}$ For example, Serra et $\mathrm{al}^{75}$ reported that IBS patients have impaired transit and altered tolerance of intestinal gas. Koide and colleagues $^{76}$ demonstrated excessive bowel gas volume among IBS subjects; however, in their symptoms did not correlate with abnormal bowel gas accumulation suggesting that gas production is not the only factor impacting symptom elicitation.

IBS patients show an altered profile of gut microbiota composition. ${ }^{77}$ Earlier studies found that the intestinal microbiota in IBS patients differs from that in healthy individuals, with a consistent decrease in the Bifidobacterium spp. population and an increase in the Enterobacter population. ${ }^{78}$ Other studies in patients with IBS have shown alterations in the microbiota, such as an increased ratio of Firmicutes to Bacteroidetes and a reduction in Lacto- 
bacillus species. ${ }^{79}$ Symptoms of IBS may be linked to those alterations. Similarly, in children with IBS the microbiome differs from normal children with a greater percentage of class gammaproteobacteria and a novel Ruminococcus-like microbe being found in the IBS patients. ${ }^{80}$ Recent research has focused on the role of gut microbiota in the pathophysiological pathway to infant colic, with numerous studies revealing differences in the gut microbiota between infants with and without colic. ${ }^{81-83}$ One study of $L$. reuteri ATCC 5573022 and two studies of $L$. reuteri DSM $17938{ }^{84,85}$ In breastfed infants with colic were effective, but a subsequent study of both breastfed and formula-fed infants with colic indicated L. reuteri DSM17938 to be ineffective. ${ }^{86}$ Thus, the effectiveness of pre- or probiotic therapies may vary depending upon the diet and other poorly defined factors including the pre-therapy microbiome composition.

Treatment trials for IBS with probiotics have had varied results which are likely explained by the diversity of study populations, antibiotics or probiotics used, and the small size of most studies. ${ }^{87}$ Treatment with the probiotic L. acidophilus SDC 2012 for 4 weeks improved symptoms scores among IBS patients when compared to placebo treatment. ${ }^{87}$ Treatment of IBS with E. coli DSM 17252 for 8 weeks was reported to dramatically reduce abdominal pain. ${ }^{88}$ L. plantarum $299 \mathrm{v}$ has shown reductions in abdominal pain in 2 intervention studies. ${ }^{89,90}$ Whorwell and colleagues reported that $B$. infantis 35624 improved pain in 437 subjects with IBS symptoms. ${ }^{91}$ Treatment with the antibiotic rifaximin appears to benefit some patients with $\operatorname{IBS}^{92}$ but the population that benefits and efficacy of antibiotic treatment remains controversial. ${ }^{93} \mathrm{~A}$ study by Jeffery and colleagues ${ }^{94}$ showed dysbiosis (e.g. increased Firmicutes/Bacteroides ratio) in a subgroup of IBS patients, while the remaining patients had normal-like microbiota composition. In that study, microbiota analysis identified several clear associations with clinical data; however a distinct subset of IBS patients with alterations in their microbiota did not correspond to IBS subtypes. It is thus possible that normal-like microbiota group might be less responsive to the agents aimed at modulation of the microbiota and individualized characterization of the GI microbiota may direct treatment strategies in the future.

The mechanisms by which altering the microbiome in the humans modifies visceral pain response remain undetermined but likely are similar to those described in in animal models of visceral pain as discussed above. In a recent study of healthy women, consumption of a mixture of probiotic bacteria in a fermented milk product affected the activity of brain regions that control the central processing of emotion and sensation. ${ }^{95}$ The in- gestion of the ferment milk product had no discernible effect on microbiota composition in the study suggesting that the effects on central nervous system were either induced by altered vagal afferent signaling or by systemic metabolic changes related to probiotic intake. ${ }^{13}$ IBS subjects have been shown to have higher concentrations of Firmicutes and decrease of Bacteroides-associated taxa which are butyrate producers. ${ }^{94}$ The abundance of Faecalibacterium species, which produce butyrate was reduced in IBS patients. ${ }^{96}$ Butyrate has been shown to decrease rectal pain perception in healthy individuals ${ }^{97}$ so it is possible that reductions in butyrate producing bacteria increases pain responses in IBS subjects.

One of the most fascinating observations used the GF animal model to evaluate the effect of the human IBS microbiome on pain responses. Crouzet et $\mathrm{al}^{98}$ inoculated GF rats with IBS fecal suspension and showed an increased visceral sensitivity in response to colorectal distension compared with GF rats inoculated with fecal suspensions from healthy control group. In the same study, GF rats inoculated with IBS microbiota showed abnormal gut fermentation with increased hydrogen excretion and sulfides production vs controls. ${ }^{98}$ This led the authors to hypothesize that hydrogen and hydrogen sulfide may be bacterial metabolites responsible for visceral hypersensitivity. ${ }^{99}$ They note that, in mice, hydrogen sulfide directly triggers visceral nociceptive behavior through sensitization and activation of T-type channels in the primary afferents. ${ }^{100}$ Further work using combinations of bacterial metabolic pathway analysis and metabolomic analysis are likely to yield an increased understanding of the underlying mechanisms by which the microbiome may alter visceral pain responses in humans.

\section{Summary}

The microbiome, gut and brain have a complex set of interactions that modulate responses to visceral pain. Various psychological, infectious and other stressors can disrupt this harmonious relationship and alter both the microbiome and visceral pain responses. Various approaches (probiotics and prebiotics) to restoring a less pathogenic microbiome appear to have promise to treat functional bowel disorders. It is possible that better management of dysbiosis during early life may prevent the development of life long changes in pain responsiveness. However, further research to better define the underlying mechanisms by which these effects are mediated is needed. This will likely lead to improved approaches for treating visceral pain by modifying the micro- 
biome or preventing chronic pain by preemptive maintenance of a healthy microbiome.

\section{References}

1. Amaral FA, Sachs D, Costa VV, et al. Commensal microbiota is fundamental for the development of inflammatory pain. Proc Natl Acad Sci USA 2008;105:2193-2197.

2. Verdú EF, Bercik P, Verma-Gandhu M, et al. Specific probiotic therapy attenuates antibiotic induced visceral hypersensitivity in mice. Gut 2006;55:182-190.

3. Rousseaux C, Thuru X, Gelot A, et al. Lactobacillus acidophilus modulates intestinal pain and induces opioid and cannabinoid receptors. Nat Med 2007;13:35-37.

4. Fraher MH, O'Toole PW, Quigley EM. Techniques used to characterize the gut microbiota: a guide for the clinician. Nat Rev Gastroenterol Hepatol 2012;9:312-322.

5. Gill SR, Pop M, Deboy RT, et al. Metagenomic analysis of the human distal gut microbiome. Science 2006;312:1355-1359.

6. Blaut M, Clavel T. Metabolic diversity of the intestinal microbiota: implications for health and disease. J Nutr 2007;137(3 suppl 2): 751S-755S.

7. Sharon G, Garg N, Debelius J, Knight R, Dorrestein Pieter C, Mazmanian Sarkis K. Specialized metabolites from the microbiome in health and disease. Cell Metab 2014;20:719-730.

8. Ohland CL, Jobin C. Microbial activities and intestinal homeostasis: a delicate balance between health and disease. Cell Mol Gastroenterol Hepatol 2015;1:28-40.

9. Borre YE, O'Keeffe GW, Clarke G, Stanton C, Dinan TG, Cryan JF. Microbiota and neurodevelopmental windows: implications for brain disorders. Trends Mol Med 2014;20:509-518.

10. Dominguez-Bello MG, Costello EK, Contreras M, et al. Delivery mode shapes the acquisition and structure of the initial microbiota across multiple body habitats in newborns. Proc Natl Acad Sci USA 2010;107:11971-11975.

11. Biasucci G, Rubini M, Riboni S, Morelli L, Bessi E, Retetangos C. Mode of delivery affects the bacterial community in the newborn gut. Early Hum Dev 2010;86(suppl 1):13-15.

12. Lupp C, Finlay BB. Intestinal microbiota. Curr Biol 2005;15: R235-R236.

13. Bezirtzoglou E, Tsiotsias A, Welling GW. Microbiota profile in feces of breast- and formula-fed newborns by using fluorescence in situ hybridization (FISH). Anaerobe 2011;17:478-482.

14. Rodríguez-Fandiño O, Hernández-Ruiz J, Schmulson M. From cytokines to toll-like receptors and beyond - current knowledge and future research needs in irritable bowel syndrome. J Neurogastroenterol Motil 2010;16:363-373.

15. El Aidy S, van Baarlen P, Derrien M, et al. Temporal and spatial interplay of microbiota and intestinal mucosa drive establishment of immune homeostasis in conventionalized mice. Mucosal Immunol 2012;5:567-579.

16. Rehman A, Sina C, Gavrilova O, et al. Nod2 is essential for temporal development of intestinal microbial communities. Gut 2011;60: 1354-1362.

17. Williams AM, Probert CSJ, Stepankova R, Tlaskalova-Hogenova
H, Phillips A, Bland PW. Effects of microflora on the neonatal development of gut mucosal $\mathrm{T}$ cells and myeloid cells in the mouse. Immunology 2006;119:470-478.

18. Thavagnanam S, Fleming J, Bromley A, Shields MD, Cardwell CR. A meta-analysis of the association between Caesarean section and childhood asthma. Clin Exp Allergy 2008;38:629-633.

19. Metsälä J, Lundqvist A, Virta LJ, Kaila M, Gissler M, Virtanen SM. Mother's and Offspring's Use of Antibiotics and Infant Allergy to Cow's Milk. Epidemiology 2013;24:303-309.

20. Salazar N, Arboleya S, Valdés L, et al. The human intestinal microbiome at extreme ages of life. Dietary intervention as a way to counteract alterations. Front Genet 2014;5:406.

21. Husebye E, Hellström PM, Midtvedt T. Intestinal microflora stimulates myoelectric activity of rat small intestine by promoting cyclic initiation and aboral propagation of migrating myoelectric complex. Dig Dis Sci 1994;39:946-956.

22. Al-Nedawi K, Mian MF, Hossain N, et al. Gut commensal microvesicles reproduce parent bacterial signals to host immune and enteric nervous systems. FASEB J 2014;29:684-695.

23. Hsiao EY, McBride SW, Hsien S, et al. Microbiota modulate behavioral and physiological abnormalities associated with neurodevelopmental disorders. Cell 2013;155:1451-1463.

24. Bailey MT, Dowd SE, Parry NMA, Galley JD, Schauer DB, Lyte M. Stressor exposure disrupts commensal microbial populations in the intestines and leads to increased colonization by Citrobacter rodentium. Infect Immun 2010;78:1509-1519.

25. Lyte M, Vulchanova L, Brown D. Stress at the intestinal surface: catecholamines and mucosa-bacteria interactions. Cell Tissue Res 2011;343:23-32.

26. Galley J, Nelson M, Yu Z, et al. Exposure to a social stressor disrupts the community structure of the colonic mucosa-associated microbiota. BMC Microbiol 2014;14:189.

27. Prins A. The brain-gut interaction: the conversation and the implications. S Afr J Clin Nutr 2011;24:S8-S14.

28. Furness JB. The enteric nervous system and neurogastroenterology. Nat Rev Gastroenterol Hepatol 2012;9:286-294.

29. Collins J, Borojevic R, Verdu EF, Huizinga JD, Ratcliffe EM. Intestinal microbiota influence the early postnatal development of the enteric nervous system. Neurogastroenterol Motil 2014;26:98107.

30. Sudo N, Chida Y, Aiba Y, et al. Postnatal microbial colonization programs the hypothalamic-pituitary-adrenal system for stress response in mice. J Physiol 2004;558(Pt 1):263-275.

31. Heijtz RD, Wang S, Anuar F, et al. Normal gut microbiota modulates brain development and behavior. Proc Natl Acd Sci USA 2011;108:3047-3052.

32. Bailey MT, Coe CL. Maternal separation disrupts the integrity of the intestinal microflora in infant rhesus monkeys. Dev Psychobiol 1999;35:146-155.

33. Barouei J, Moussavi M, Hodgson DM. Effect of maternal probiotic intervention on HPA axis, immunity and gut microbiota in a rat model of irritable bowel syndrome. PLoS One 2012;7:e46051.

34. Al-Chaer ED, Traub RJ. Biological basis of visceral pain: recent developments. Pain 2002;96:221-225.

35. Jones MP, Dilley JB, Drossman D, Crowell MD. Brain-gut connections in functional GI disorders: anatomic and physiologic rela- 
tionships. Neurogastroenterol Motil 2006;18:91-103.

36. Sengupta J. Visceral Pain: The neurophysiological mechanism. In: Canning BJ, Spina D, eds. Sensory nerves. Berlin Heidelberg: Springer 2009:31-74.

37. Coutinho SV, Plotsky PM, Sablad M, et al. Neonatal maternal separation alters stress-induced responses to viscerosomatic nociceptive stimuli in rat. Am J Physiol Gastrointest Liver Physiol 2002;282: G307-G316.

38. Miranda A, Peles S, Rudolph C, Shaker R, Sengupta JN. Altered visceral sensation in response to somatic pain in the rat. Gastroenterology 2004;126:1082-1089.

39. Smith C, Nordstrom E, Sengupta JN, Miranda A. Neonatal gastric suctioning results in chronic visceral and somatic hyperalgesia: role of corticotropin releasing factor. Neurogastroenterol Motil 2007;19: 692-699.

40. Johnson A, Greenwood-Van Meerveld B, McRorie J. Effects of Bifidobacterium infantis 35624 on post-inflammatory visceral hypersensitivity in the rat. Dig Dis Sci 2011;56:3179-3186.

41. Desbonnet L, Garrett L, Clarke G, Kiely B, Cryan JF, Dinan TG. Effects of the probiotic Bifidobacterium infantis in the maternal separation model of depression. Neuroscience 2010;170:1179-1188.

42. Ma X, Mao Y-K, Wang B, Huizinga JD, Bienenstock J, Kunze W. Lactobacillus reuteri ingestion prevents hyperexcitability of colonic DRG neurons induced by noxious stimuli. Am J Physiol Gastrointest Liver Physiol 2009;296:G868-875.

43. McKernan DP, Fitzgerald P, Dinan TG, Cryan JF. The probiotic Bifidobacterium infantis 35624 displays visceral antinociceptive effects in the rat. Neurogastroenterol Motil 2010;22:1029-1035, e268.

44. Belkaid Y, Hand TW. Role of the microbiota in immunity and inflammation. Cell 2014;157:121-141.

45. Gadotti VM, Martins DF, Pinto HF, et al. Diacerein decreases visceral pain through inhibition of glutamatergic neurotransmission and cytokine signaling in mice. Pharmacol Biochem Behav 2012;102: 549-554.

46. Aguilera M, Cerdà-Cuéllar M, Martínez V. Antibiotic-induced dysbiosis alters host-bacterial interactions and leads to colonic sensory and motor changes in mice. Gut Microbes 2015;6:10-23.

47. Hughes PA, Zola H, Penttila IA, Blackshaw LA, Andrews JM, Krumbiegel D. Immune activation in irritable bowel syndrome: can neuroimmune interactions explain symptoms? Am J Gastroenterol 2013;108:1066-1074.

48. Kunze WA, Mao Y-K, Wang B, et al. Lactobacillus reuteri enhances excitability of colonic $\mathrm{AH}$ neurons by inhibiting calcium- dependent potassium channel opening. J Cell Mol Med 2009;13:2261-2270.

49. Wang B, Mao Yk, Diorio C, et al. Lactobacillus reuteri ingestion and IKCa channel blockade have similar effects on rat colon motility and myenteric neurones. Neurogastroenterol Motil 2010;22:98-107, e33.

50. Ait-belgnaoui A, Eutamene H, Houdeau E, Bueno L, Fioramonti J, Theodorou V. Lactobacillus farciminis treatment attenuates stress-induced overexpression of Fos protein in spinal and supraspinal sites after colorectal distension in rats. Neurogastroenterol Motil 2009;21: 567-573, e18-e19.

51. O'Mahony L, McCarthy J, Kelly P, et al. Lactobacillus and Bifidobacterium in irritable bowel syndrome: Symptom responses and relationship to cytokine profiles. Gastroenterology 2005;128:541-551.

52. Bravo JA, Forsythe P, Chew MV, et al. Ingestion of Lactobacillus strain regulates emotional behavior and central GABA receptor expression in a mouse via the vagus nerve. Proc Natl Acad Sci USA 2011;108:16050-16055.

53. Bercik P, Park AJ, Sinclair D, et al. The anxiolytic effect of Bifidobacterium longum NCC3001 involves vagal pathways for gutbrain communication. Neurogastroenterol Motil 2011;23:1132-1139.

54. Bandlapalli P, Banji D, Banji OJF, Swetha D, Pratusha NG. Role of stress in pathophysiology of irritable bowel syndrome. IRJP 2011;2: 54-60.

55. Taché Y, Martinez V, Million M, Wang L. Stress and the gastrointestinal trat III. Stress-related alterations of gut motor function: role of brain corticotropin-releasing factor receptors. Am J Physiol Gastrointest Liver Physiol 2001;280:G173-G177.

56. Aguilera M, Vergara P, Martínez V. Stress and antibiotics alter luminal and wall-adhered microbiota and enhance the local expression of visceral sensory-related systems in mice. Neurogastroenterol Motil 2013;25:e515-e529.

57. O'Mahony SM, Felice VD, Nally K, et al. Disturbance of the gut microbiota in early-life selectively affects visceral pain in adulthood without impacting cognitive or anxiety-related behaviors in male rats. Neuroscience 2014;277:885-901.

58. Kannampalli P, Pochiraju S, Chichlowski M, et al. Probiotic Lactobacillus rhamnosus GG (LGG) and prebiotic prevent neonatal inflammation-induced visceral hypersensitivity in adult rats. Neurogastroenterol Motil 2014;26:1694-1704.

59. Marks DM, Shah MJ, Patkar AA, Masand PS, Park GY, Pae CU Serotonin-norepinephrine reuptake inhibitors for pain control: premise and promise. Curr Neuropharmacol 2009;7:331-336.

60. Kamiya T, Wang L, Forsythe P, et al. Inhibitory effects of Lactobacillus reuteri on visceral pain induced by colorectal distension in SpragueDawley rats. Gut 2006;5 5:191-196.

61. O'Mahony SM, Marchesi JR, Scully P, et al. Early life stress alters behavior, immunity, and microbiota in rats: implications for irritable bowel syndrome and psychiatric illnesses. Biol Psychiatry 2009;65: 263-267.

62. DuPont AW, DuPont HL. The intestinal microbiota and chronic disorders of the gut. Nat Rev Gastroenterol Hepatol 2011;8:523531.

63. Rutten JMTM, Benninga MA, Vlieger AM. IBS and FAPS in children: a comparison of psychological and clinical characteristics. J Pediatr Gastroenterol Nutr 2014;59:493-499.

64. Quigley EM. Changing face of irritable bowel syndrome. World J Gastroenterol 2006;12:1-5.

65. Tanaka Y, Kanazawa M, Fukudo S, Drossman DA. Biopsychosocial model of irritable bowel syndrome. J Neurogastroenterol Motil 2011;17:131-139.

66. Barbara G, De Giorgio R, Stanghellini V, Cremon C, Salvioli B, Corinaldesi R. New pathophysiological mechanisms in irritable bowel syndrome. Aliment Pharmacol Ther 2004;20(suppl 2):1-9.

67. Walker EA, Gelfand AN, Gelfand MD, Green C, Katon WJ. Chronic pelvic pain and gynecological symptoms in women with irritable bowel syndrome. J Psychosom Obstet Gynaecol 1996;17:39- 46.

68. Mayer EA, Berman S, Suyenobu B, et al. Differences in brain responses to visceral pain between patients with irritable bowel syndrome and ulcerative colitis. Pain 2005;115:398-409.

69. Mertz H, Morgan V, Tanner G, et al. Regional cerebral activation 
in irritable bowel syndrome and control subjects with painful and nonpainful rectal distention. Gastroenterology 2000;118:842-848.

70. Walker LS, Garber J, Smith CA, Van Slyke DA, Claar RL. The relation of daily stressors to somatic and emotional symptoms in children with and without recurrent abdominal pain. J Consult Clin Psychol 2001;69:85-91.

71. Awad RA, Camacho S, Martín J, Ríos N. Rectal sensation, pelvic floor function and symptom severity in Hispanic population with irritable bowel syndrome with constipation. Colorectal Dis 2006;8: 488-493.

72. Dickhaus B, Mayer EA, Firooz N, et al. Irritable bowel syndrome patients show enhanced modulation of visceral perception by auditory stress. Am J Gastroenterol 2003;98:135-143.

73. Posserud I, Agerforz P, Ekman R, Björnsson ES, Abrahamsson H, Simrén M. Altered visceral perceptual and neuroendocrine response in patients with irritable bowel syndrome during mental stress. Gut 2004;53:1102-1108.

74. Agrawal A, Whorwell PJ. Review article: abdominal bloating and distension in functional gastrointestinal disorders-epidemiology and exploration of possible mechanisms. Aliment Pharmacol Ther 2008; 27:2-10.

75. Serra J, Azpiroz F, Malagelada JR. Impaired transit and tolerance of intestinal gas in the irritable bowel syndrome. Gut 2001;48:14-19.

76. Koide A, Yamaguchi T, Odaka T, et al. Quantitative analysis of bowel gas using plain abdominal radiograph in patients with irritable bowel syndrome. Am J Gastroenterol 2000;95:1735-1741.

77. Kassinen A, Krogius-Kurikka L, Mäkivuokko H, et al. The fecal microbiota of irritable bowel syndrome patients differs significantly from that of healthy subjects. Gastroenterology. 2007;133:24-33.

78. Si JM, Yu YC, Fan YJ, Chen SJ. Intestinal microecology and quality of life in irritable bowel syndrome patients. World J Gastroenterol 2004;10:1802-1805.

79. Rajilić-Stojanović M, Biagi E, Heilig HGHJ, et al. Global and deep molecular analysis of microbiota signatures in fecal samples from patients with irritable bowel syndrome. Gastroenterology 2011;141: 1792-1801.

80. Saulnier DM, Riehle K, Mistretta TA, et al. Gastrointestinal Microbiome signatures of pediatric patients with irritable bowel syndrome. Gastroenterology 2011;141:1782-1791.

81. Lehtonen L, Korvenranta H, Eerola E. Intestinal microflora in colicky and noncolicky infants: bacterial cultures and gas-liquid chromatography. J Pediatr Gastroenterol Nutr 1994;19:310-314.

82. Mentula S, Tuure T, Koskenala R, Korpela R, Könönen E. Microbial composition and fecal fermentation end products from colicky infants - a probiotic supplementation pilot. Microb Ecol Health Dis 2008;20:37-47.

83. Pärtty A, Kalliomäki M, Endo A, Salminen S, Isolauri E. Compositional development of Bifidobacterium and Lactobacillus microbiota is linked with crying and fussing in early infancy. PLoS One 2012;7: e32495.

84. Savino F, Cordisco L, Tarasco V, et al. Lactobacillus reuteri DSM 17938 in infantile colic: a randomized, double-blind, placebo-con- trolled trial. Pediatrics 2010;126:e526-e533.

85. Szajewska H, Gyrczuk E, Horvath A. Lactobacillus reuteri DSM 17938 for the management of infantile colic in breastfed infants: a randomized, double-blind, placebo-controlled trial. J Pediatr 2013; 162:257-262.

86. Sung V, Hiscock $H$, Tang ML, et al. Treating infant colic with the probiotic Lactobacillus reuteri: double blind, placebo controlled randomised trial 2014;348:g2107.

87. Sinn D, Song J, Kim HJ, et al. Therapeutic effect of Lactobacillus acidophilus-SDC 2012, 2013 in patients with irritable bowel syndrome. Dig Dis Sci 2008;53:2714-2718.

88. Enck P, Zimmermann K, Menke G, Klosterhalfen S. Randomized controlled treatment trial of irritable bowel syndrome with a probiotic E-coli preparation (DSM17252) compared to placebo. Z Gastroenterol 2009;47:209-214.

89. Nobaek S, Johansson ML, Molin G, Ahrné S, Jeppsson B. Alteration of intestinal microflora is associated with reduction in abdominal bloating and pain in patients with irritable bowel syndrome. Am J Gastroenterol 2000;95:1231-1238.

90. Niedzielin K, Kordecki H, Birkenfeld B. A controlled, doubleblind, randomized study on the efficacy of Lactobacillus plantarum $299 \mathrm{~V}$ in patients with irritable bowel syndrome. Eur J Gastroenterol Hepatol 2001;13:1143-1147.

91. Whorwell PJ, Altringer L, Morel J, et al. Efficacy of an encapsulated probiotic Bifidobacterium infantis 35624 in women with irritable bowel syndrome. Am J Gastroenterol 2006;101:1581-1590.

92. Pimentel M, Lembo A, Chey WD, et al. Rifaximin therapy for patients with irritable bowel syndrome without constipation. New Engl J Med 2011;364:22-32.

93. Saadi M, McCallum RW. Rifaximin in irritable bowel syndrome: rationale, evidence and clinical use. Ther Adv Chronic Dis 2013;4: 71-75.

94. Jeffery IB, O'Toole PW, Öhman L, et al. An irritable bowel syndrome subtype defined by species-specific alterations in faecal microbiota. Gut 2012;61:997-1006.

95. Tillisch K, Labus J, Kilpatrick L, et al. Consumption of fermented milk product with probiotic modulates brain activity. Gastroenterology 2013;144:1394-1401, e1-e4.

96. Duncan SH, Holtrop G, Lobley GE, Calder AG, Stewart CS, Flint $\mathrm{HJ}$. Contribution of acetate to butyrate formation by human faecal bacteria. Br J Nutr 2004;91:915-923.

97. Vanhoutvin SA, Troost FJ, Kilkens TO, et al. The effects of butyrate enemas on visceral perception in healthy volunteers. Neurogastroenterol Motil 2009;21:952-e76.

98. Crouzet L, Gaultier E, Del'Homme C, et al. The hypersensitivity to colonic distension of IBS patients can be transferred to rats through their fecal microbiota. Neurogastroenterol Motil 2013;25:e272-e282.

99. King TS, Elia M, Hunter JO. Abnormal colonic fermentation in irritable bowel syndrome. Lancet 1998;352:1187-1189.

100. Matsunami M, Tarui T, Mitani K, et al. Luminal hydrogen sulfide plays a pronociceptive role in mouse colon. Gut 2009;58:751-761. 